\title{
The effect of $\mathbf{R}$ parity violating couplings on $e^{-} e^{-} \rightarrow \tilde{e}_{L} \tilde{e}_{R}$
}

\author{
Uma Mahanta \\ Mehta Research Institute \\ Chhatnag Road, Jhusi \\ Allahabad-211019, India
}

\begin{abstract}
In this work we study the effect of $\mathrm{R}$ parity breaking couplings on the fermion number violating process $e^{-} e^{-} \rightarrow \tilde{e}_{L} \tilde{e}_{R}$. We find that an $e^{-} e^{-}$linear collider operating at $\sqrt{s}=$ $500 \mathrm{Gev}$ with an integrated luminosity of $50 \mathrm{fb}^{-1}$ will be able to probe the coupling $\left(\lambda_{211}^{2}+\lambda_{311}^{3}\right)^{\frac{1}{2}}$ down to .045 for a bino mass of $100 \mathrm{Gev}$ and selectron mass of $200 \mathrm{Gev}$. This would improve the present bound on it by a factor of 3.5. More improved bounds can be obtained from precision measurement of $\sigma_{L R}-\sigma_{R R}$ which reduces the MSSM background.
\end{abstract}


The process $e^{-} e^{-} \rightarrow \tilde{e}_{L} \tilde{e}_{R}$ violates fermion number by two units. In the context of MSSM [1] with conserved R parity it occurs in the lowest order via the $t$ channel exchange of a bino which we shall assume to be the lightest neutralino in our study. The fermion number violation occurs because the $\tilde{B}$ being a Majorana fermion contributes a fermion number violating propagator to the transition matrix element. Suppose the MSSM Lagrangian is now extended by the $\mathrm{R}$ parity violating term that involves the product of three lepton superfields [2]. The process $e^{-} e^{-} \rightarrow \tilde{e}_{L} \tilde{e}_{R}$ then receives an additional contribution from thr $\mathrm{t}$ channel exchange of a $\nu_{\mu}$ or $\nu_{\tau}$ even if they are assumed to be Dirac fermions having only fermion number conserving propagators. The fermion number violation in this case arises from the $\mathrm{R}$ parity breaking term that violates fermion number precisely by two units.

Consider the part of the MSSM Lagrangian that gives the interaction of $\tilde{B}$ and $\tilde{W}_{3}$ with the $e-\tilde{e}$ pair [1].

$$
L_{0}=\frac{1}{\sqrt{2}}\left[\bar{e}_{R}\left(g \tilde{W}_{3}+g^{\prime} \tilde{B}\right) \tilde{e}_{L}+2 g^{\prime} \bar{e}_{L} \tilde{B} \tilde{e}_{R}\right]+\text { h.c. }
$$

Among $\tilde{B}$ and $\tilde{W}_{3}$, only $\tilde{B}$ interacts both with $\tilde{e}_{L}$ and $\tilde{e}_{R}$. In the context of MSSM the process $e^{-} e^{-} \rightarrow \tilde{e}_{L} \tilde{e}_{R}$ can therefore occur through the t channel exchange of $\tilde{B}$ only. The cross section for the process $e^{-} e^{-} \rightarrow \tilde{e}_{L} \tilde{e}_{R}$ was first computed by Keung and Littenburg [3]. The matrix elements for t channel and u channel exchange of $\tilde{B}$ are given by

$$
M_{a}=g^{\prime 2} \bar{v}\left(p_{2}, s_{2}\right) \frac{\left(p_{1}-k_{1}\right) \cdot \gamma}{\left(t-M^{2}\right)} P_{L} u\left(p_{1}, s_{1}\right) .
$$

and

$$
M_{b}=g^{\prime 2} \bar{v}\left(p_{2}, s_{2}\right) \frac{\left(p_{1}-k_{2}\right) \cdot \gamma}{\left(u-M^{2}\right)} P_{R} u\left(p_{1}, s_{1}\right) .
$$

In the above $\left(p_{1}, s_{1}\right)$ and $\left(p_{2}, s_{2}\right)$ are the momenta and spins of the two incoming electrons. $k_{1}$ and $k_{2}$ are the momenta of the outgoing $\tilde{e}_{L}$ and $\tilde{e}_{R}$ respectively. M is the mass 
of $\tilde{B} . t=\left(p_{1}-k_{1}\right)^{2}=\left(p_{2}-k_{2}\right)^{2}$ and $u=\left(p_{1}-k_{2}\right)^{2}=\left(p_{2}-k_{1}\right)^{2} \cdot\left(p_{1}-k_{1}\right) \cdot \gamma=\left(p_{1}-k_{1}\right)^{\mu} \gamma_{\mu}$ and $\left(p_{1}-k_{2}\right) \cdot \gamma=\left(p_{1}-k_{2}\right)^{\mu} \gamma_{\mu}$. To simplify our analysis we shall assume that the mass of $\tilde{e}_{L}$ and $\tilde{e}_{R}$ are equal and we shall denote their common mass by $\mathrm{m}$.

If the incoming electron beams are both unpolarized then the total matrix element for the process is given by $M=M_{a}+M_{b}$. The differential scattering cross-section for the MSSM contribution is given by

$$
\frac{d \sigma_{0}}{d y}=\frac{1}{128 \pi s} g^{\prime 4}\left(1-a-y^{2}\right)\left[\frac{1}{(1-b-y)^{2}}+\frac{1}{(1-b+y)^{2}}\right] .
$$

Here $y=\beta x=\left(1-4 \frac{m^{2}}{s}\right)^{\frac{1}{2}} \cos \theta, a=\frac{m^{2}}{s}$ and $b=\frac{m^{2}-M^{2}}{s} . \sigma_{0}$ is the MSSM contribution to the cross section. Note that in the MSSM contribution to $e^{-} e^{-} \rightarrow \tilde{e}_{L} \tilde{e}_{R}$ there is no interference between t channel and $u$ channel exchanges.

Let us now consider the MSSM Lagrangian to be extended by the following R parity violating terms

$$
L_{1}=\left[\lambda_{211}\left\{\tilde{e}_{L} \bar{e}_{R} \nu_{\mu L}+\tilde{e}_{R}^{*}\left(\bar{\nu}_{\mu L}\right)^{c} e_{L}\right\}+\lambda_{311}\left\{\tilde{e}_{L} \bar{e}_{R} \nu_{\tau L}+\tilde{e}_{R}^{*}\left(\bar{\nu}_{\tau L}\right)^{c} e_{L}\right\}\right]+\text { h.c. }
$$

The process $e^{-} e^{-} \rightarrow \tilde{e}_{L} \tilde{e}_{R}$ violates fermion number by two units but it conserves $\mathrm{R}$ parity . The transition matrix element should therefore involve an even number of R parity violating interaction vertices. In the above Lagrangian the term $\lambda_{211} \tilde{e}_{R}^{*}\left(\bar{\nu}_{\mu L}\right)^{c} e_{L}$ violates fermion number by two units but the term $\lambda_{211}^{*} \tilde{e}_{L}^{*} \bar{\nu}_{\mu L} e_{R}$ conserves fermion number. Hence the product of $\lambda_{211} \tilde{e}_{R}^{*}\left(\bar{\nu}_{\mu L}\right)^{c} e_{L}$ and $\lambda_{211}^{*} \tilde{e}_{L}^{*} \bar{\nu}_{\mu L} e_{R}$ which occurs in second order perturbation expansion can contribute to $e^{-} e^{-} \rightarrow \tilde{e}_{L} \tilde{e}_{R}$. This also holds true for analogous terms proportional to $\lambda_{311}$ and involving $\nu_{\tau}$. Note that $\left(\lambda_{211} \tilde{e}_{R}^{*}\left(\bar{\nu}_{\mu L}\right)^{c} e_{L}\right)^{2}$ or $\left(\lambda_{311} \tilde{e}_{R}^{*}\left(\bar{\nu}_{\tau L}\right)^{c} e_{L}\right)^{2}$ which also occur in second order pertubation expansion cannot contribute to the process $e^{-} e^{-} \rightarrow \tilde{e}_{R} \tilde{e}_{R}$ if the neutrinos are assumed to be Dirac fermions having only fermion number conserving propagators. Similarly $\left(\lambda_{211} \tilde{e}_{L}^{*} \bar{\nu}_{\mu L} e_{R}\right)^{2}$ or $\left(\lambda_{311} \tilde{e}_{L}^{*} \bar{\nu}_{\tau L} e_{R}\right)^{2}$ cannot contribute to the process $e^{-} e^{-} \rightarrow \tilde{e}_{L} \tilde{e}_{L}$. Hence $\mathrm{R}$ parity violating couplings affect only the 
process $e^{-} e^{-} \rightarrow \tilde{e}_{L} \tilde{e}_{R}$ leaving $e^{-} e^{-} \rightarrow \tilde{e}_{L} \tilde{e}_{L}$ and $e^{-} e^{-} \rightarrow \tilde{e}_{R} \tilde{e}_{R}$ unchanged.

We shall now evaluate the transition matrix elements arising from $\mathrm{R}$ parity violating couplings. Consider first the t channel exchange of a $\nu_{\mu}$. We have

$$
\begin{aligned}
-i \delta M_{a}^{\mu} & =-\left|\lambda_{211}\right|^{2}<\tilde{e}_{R}\left(k_{2}\right) \tilde{e}_{L}\left(k_{1}\right)\left|\tilde{e}_{R}^{*}\left(\bar{\nu}_{\mu L}\right)^{c} e_{L} \tilde{e}_{L}^{*} \bar{\nu}_{\mu L} e_{R}\right| e\left(p_{1}, s_{1}\right) ; e\left(p_{2}, s_{2}\right)> \\
& =\left|\lambda_{211}\right|^{2}<0\left|\nu_{\mu}^{T} C^{+} P_{L} e \bar{\nu}_{\mu} P_{R} e\right| e\left(p_{1}, s_{1}\right) e\left(p_{2}, s_{2}\right)> \\
& =-\left|\lambda_{211}\right|^{2} u^{T}\left(p_{2}, s_{2}\right) P_{L}^{T} C^{+T}<0\left|T\left\{\nu_{\mu} \bar{\nu}_{\mu}\right\}\right| 0>P_{R} u\left(p_{1}, s_{1}\right) \\
& =\left|\lambda_{211}\right|^{2} \bar{v}\left(p_{2}, s_{2}\right) C P_{L}^{T} C^{+T} \frac{i\left(p_{1}-k_{1}\right) \cdot \gamma}{\left(p_{1}-k_{1}\right)^{2}} P_{R} u\left(p_{1}, s_{1}\right) \\
& =-i\left|\lambda_{211}\right|^{2} \bar{v}\left(p_{2}, s_{2}\right) \frac{\left(p_{1}-k_{1}\right) \cdot \gamma}{t} P_{R} u\left(p_{1}, s_{1}\right) .
\end{aligned}
$$

Adding the contribution due to t channel exchange of $\nu_{\tau}$ we get

$$
\delta M_{a}=\frac{\left|\lambda_{211}\right|^{2}+\left|\lambda_{311}\right|^{2}}{t} \bar{v}\left(p_{2}, s_{2}\right)\left(p_{1}-k_{1}\right) \cdot \gamma P_{R} u\left(p_{1}, s_{1}\right)
$$

Consider next the matrix element due to $u$ channel exchange of $\nu_{\mu}$. We have

$$
\begin{aligned}
-i \delta M_{b}^{\mu} & =-\left|\lambda_{211}\right|^{2}<\tilde{e}_{R}\left(k_{2}\right) \tilde{e}_{L}\left(k_{1}\right)\left|\tilde{e}_{L}^{*} \bar{\nu}_{\mu L} e_{R} \tilde{e}_{R}^{*}\left(\bar{\nu}_{\mu L}\right)^{c} e_{L}\right| e\left(p_{1}, s_{1}\right) ; e\left(p_{2}, s_{2}\right)> \\
& =\left|\lambda_{211}\right|^{2}<0\left|\bar{\nu}_{\mu} P_{R} e \nu_{\mu}^{T} C^{+} P_{L} e\right| e\left(p_{1}, s_{1}\right) ; e\left(p_{2}, s_{2}\right)> \\
& =-\left|\lambda_{211}\right|^{2} u^{T}\left(p_{2}, s_{2}\right) P_{R}^{T}<0\left|T\left\{\bar{\nu}_{\mu}^{T} \nu_{\mu}^{T}\right\}\right| 0>C^{+} P_{L} u\left(p_{1}, s_{1}\right) \\
& =\left|\lambda_{211}\right|^{2} u^{T}\left(p_{2}, s_{2}\right) P_{R}^{T}<0\left|T\left\{\nu_{\mu} \bar{\nu}_{\mu}\right\}^{T}\right| 0>C^{+} P_{L} u\left(p_{1}, s_{1}\right) \\
& =-\left|\lambda_{211}\right|^{2} \bar{v}\left(p_{2}, s_{2}\right) C P_{R}^{T} \frac{i\left(p_{2}-k_{1}\right) \cdot \gamma^{T}}{u} C^{+} P_{L} u\left(p_{1}, s_{1}\right) \\
& =-i \frac{\left|\lambda_{211}\right|^{2}}{u} \bar{v}\left(p_{2}, s_{2}\right)\left(p_{1}-k_{2}\right) \cdot \gamma P_{L} u\left(p_{1}, s_{1}\right) .
\end{aligned}
$$

Adding the contribution of $\nu_{\tau}$ we get

$$
\delta M_{b}=\frac{\left|\lambda_{211}\right|^{2}+\left|\lambda_{311}\right|^{2}}{u} \bar{v}\left(p_{2}, s_{2}\right)\left(p_{1}-k_{2}\right) \cdot \gamma P_{L} u\left(p_{1}, s_{1}\right) .
$$


Therefore the total matrix element arising from $\mathrm{R}$ parity violating couplings is given by

$$
\delta M=\left(\left|\lambda_{211}\right|^{2}+\left|\lambda_{311}\right|^{2}\right) \bar{v}\left(p_{2}, s_{2}\right)\left[\frac{\left(p_{1}-k_{1}\right) \cdot \gamma}{t} P_{R}+\frac{\left(p_{1}-k_{2}\right) \cdot \gamma}{u} P_{L}\right] u\left(p_{1}, s_{1}\right)
$$

In deriving the matrix elements due to $\mathrm{R}$ parity violating couplings particular care has been taken in transposing a pair of Fermi fields because of their anticommuting nature.

The differential scattering cross section due to MMSM plus R parity violating couplings is given by

$$
\begin{aligned}
\frac{d \sigma_{t}}{d y} & =\frac{g^{\prime 4}}{128 \pi s}\left[\left(1-a-y^{2}\right)\left\{\frac{1}{(1-b-y)^{2}}+\frac{1}{(1-b+y)^{2}}\right\}\right. \\
& +\frac{\lambda^{4}}{g^{\prime 4}}\left(1-a-y^{2}\right)\left\{\frac{1}{(1-a-y)^{2}}+\frac{1}{(1-a+y)^{2}}\right\} \\
& \left.+\frac{\lambda^{2}}{g^{\prime 2}}\left(1+2 a+2 y^{2}\right)\left\{\frac{1}{(1-a+y)(1-b-y)}+\frac{1}{(1-a-y)(1-b+y)}\right\}\right]
\end{aligned}
$$

where $\lambda^{2}=\left[\lambda_{211}^{2}+\lambda_{311}^{2}\right]$.

In MSSM the lightest neutralino usually also happens to be the lightest supersymmetric particle (LSP). For a bino mass of $100 \mathrm{Gev}$, selectron mass of $200 \mathrm{Gev}$ and $\sqrt{s}=500$ Gev we find that $\sigma_{0}=240.82 \mathrm{fb}$ and $\sigma_{t}=\sigma_{0}+\delta \sigma=\left(240.82+3117.8 \lambda^{2}+16998.7 \lambda^{4}\right) \mathrm{fb}$. A bound on $\lambda$ can be derived by requiring that for observability, the signal $\delta \sigma \int L d t$ should be at least greater than or equal to $3\left[\sigma_{0} \int L d t\right]^{\frac{1}{2}}$. For an $e^{-} e^{-}$linear collider operating at $\sqrt{s}=500 \mathrm{Gev}$ with an integrated luminosity of of $50 \mathrm{fb}^{-1}$ this produces the bound $\lambda \leq$ .045. The present best bound on $\lambda$ for $\mathrm{m}=200 \mathrm{Gev}$ is .156 [4]. The bound obtainable from an $e^{-} e^{-}$collider under the above conditions will therefore improve the present bound by a factor of 3.5. The present bounds on $\mathrm{R}$ parity breaking couplings which are mostly obtained from low energy measurements scale with $\mathrm{m}$. Hence by choosing higher values of $\sqrt{s}$ which would enable the production of heavier selectrons, the collider bound can be 
much smaller than the present bound. In some supersymmetric models as for example in scenarios with gauge mediated supersymmetry breaking the selectron often turns out to be the LSP. For a selectron mass of $150 \mathrm{Gev}$, bino mass of $250 \mathrm{Gev}$ and $\sqrt{s}=500 \mathrm{Gev}$ we find that $\sigma_{0}=173.69 \mathrm{fb}$ and $\delta \sigma=\left(3718.72 \lambda^{2}+31478.2 \lambda^{4}\right) \mathrm{fb}$. Under the same operating conditions as above $\left(\sqrt{s}=500 \mathrm{Gev}\right.$ and $\int L d t=50 \mathrm{fb}^{-1}$ we obtain the bound $\lambda \leq .039$. This should be compared with the present bound of .117 for $\mathrm{m}=150 \mathrm{Gev}$. More improved bound on $\lambda$ can be derived from precision measurement of $\sigma_{L R}-\sigma_{R R}$ where $\sigma_{L R}$ is the cross section for $e^{-} e^{-} \rightarrow \tilde{e}_{L} \tilde{e}_{R}$ and $\sigma_{R R}$ is that of $e^{-} e^{-} \rightarrow \tilde{e}_{L} \tilde{e}_{R}$. Since $\mathrm{R}$ parity violating couplings affect only $\sigma_{L R}$ the MSSM background for $\sigma_{L R}-\sigma_{R R}$ will be lower than that for $\sigma_{L R}$. The reduction of MSSM background will however be significant only if the bino mass is not too small compared to $\sqrt{s}$. The reason being $\sigma_{R R}$ receives contribution from the chirality flipping part of the bino propagator and is proportional to $\frac{M^{2}}{\mathrm{~s}}$. For $\mathrm{m}=150$ Gev, $\mathrm{M}=250 \mathrm{G}$ ev and $\sqrt{s}=500 \mathrm{Gev}$ we find that $\sigma_{R R}=241.01 \mathrm{fb}$. Hence under the same operating conditions as before, from precision measurement of $\sigma_{L R}-\sigma_{R R}$ it should be possible to probe $\lambda$ down to .030 .

In conclusion in this work we have studied the effect of $\mathrm{R}$ parity breaking couplings on the fermion number violating process $e^{-} e^{-} \rightarrow \tilde{e}_{L} \tilde{e}_{R}$. We find that an $e^{-} e^{-}$linear collider operating at $\sqrt{s}=500 \mathrm{Gev}$ with an integrated luminosity of $50 \mathrm{fb}^{-1}$ should be able to probe the coupling $\left[\lambda_{211}^{2}+\lambda_{311}^{2}\right]^{\frac{1}{2}}$ down to .045 for $\mathrm{M}=100 \mathrm{Gev}$ and $\mathrm{m}=200 \mathrm{Gev}$. This bound can be obtained simply from precision measurement of the total cross section $\sigma_{L R}$. More improved bounds can be derived from precision measurement of $\sigma_{L R}-\sigma_{R R}$ which reduces the MSSM background. The $e^{-} e^{-}$mode of a linear collider has recently been proposed [5] for high precision measurement of superparticle couplings and verfying the supersymmetric relation between ordinary couplings and superparticle couplings. Our study clearly shows that to measure bino couplings precisely one should use the process $e^{-} e^{-} \rightarrow \tilde{e}_{R} \tilde{e}_{R}$ instead of $e^{-} e^{-} \rightarrow \tilde{e}_{L} \tilde{e}_{R}$ since the former as opposed to the later is not affected even if $\mathrm{R}$ parity violating couplings are present. 


\section{References}

1. H. P. Nilles, Phys. Rep. 110, 1 (1984); H. E. Haber and G. L. Kane, Phys. Rep. 117, $75,(1985)$.

2. F. Zwirner, Phys. Lett. B 132, 103 (1983); L. J. Hall and M. Suzuki, Nucl. Phys. B 231, 419 (1984); G. G. Ross and J. W. F. Valle, Phys. Lett. B 151, 375 (1985); S. Dawson, Nucl. Phys. B 261, 297 (1985); V. Barger, G. F. Giudice and T. Han, Phys. Rev. D 40, 2987 (1989).

3. W. Y. Keung and L. Littenburg, Phys. Rev. D 28, 1067 (1983); H. E. Haber and G. L. Kane, Phys. Rep. 117, 75 (1985).

4. H. Dreiner, hep-ph/9707435.

5. H. C. Cheng, J. L. Feng and N. Polonsky, Phys. Rev. D 57, 152 (1998); H. C. Cheng, hep-ph/9801234. 